\title{
Reduction of ischemic spinal cord injury by dextrorphan: Comparison of several methods of administration
}

Hitoshi Terada, MD

Teruhisa Kazui, MD

Makoto Takinami, MD

Katsushi Yamashita, MD

Naoki Washiyama, MD

Bashar Abul Hasan Muhammad, MBBS
From the First Department of Surgery, Hamamatsu University School of Medicine, Hamamatsu, Japan.

Received for publication Dec 14, 2000; revisions requested Jan 22, 2001; revisions received Feb 6, 2001; accepted for publication May 16, 2001.

Address for reprints: Hitoshi Terada, MD, First Department of Surgery, Hamamatsu University School of Medicine, 3600, Handa-cho, Hamamatsu, 431-3192, Japan (E-mail: terada@hama-med.ac.jp).

J Thorac Cardiovasc Surg 2001;122:979-85

Copyright @ $\odot 2001$ by The American Association for Thoracic Surgery

$0022-5223 / 2001 \$ 35.00+0 \quad \mathbf{1 2 / 1 / 1 1 7 2 7 8}$

doi:10.1067/mtc.2001.117278
Objectives: We investigated the effect of dextrorphan, an $N$-methyl-D-aspartate receptor antagonist, on the reduction of ischemic spinal cord injury and the safe clamping time after various methods of administration.

Methods: Spinal cord ischemia was induced in New Zealand White rabbits by infrarenal aortic clamping and animals were divided into 5 groups. Group A ( $\mathrm{n}=$ $15)$ received simple clamping. Groups $B(n=20)$ and $C(n=35)$ received dextrorphan pretreatment $(10 \mathrm{mg} / \mathrm{kg})$, followed by continuous intravenous or intra-aortic infusion $(1 \mathrm{mg} / \mathrm{min})$, respectively. Group $\mathrm{D}(\mathrm{n}=25)$ received the same dextrorphan pretreatment and bolus intra-aortic injection at clamping ( $1 \mathrm{mg}$ per minute of clamping time). Group E $(n=15)$ received bolus intrathecal injection of dextrorphan $(0.2 \mathrm{mg} / \mathrm{kg})$. Each dextrorphan-treated group had a small group of control animals $(n=5)$. The neurologic status was assessed by the Johnson score $(5=$ normal, $0=$ paraplegic) 48 hours after unclamping, and animals were put to death for histopathologic examination.

Results: All dextrorphan-treated groups showed better neurologic function than the respective control animals $(P<.001$ vs groups $\mathrm{B}, \mathrm{C}$, and $\mathrm{D} ; P=.014$ vs group $\mathrm{E})$. The order of efficacy of dextrorphan (as revealed by the average of neurologic status) was as follows: group $\mathrm{C}>$ group $\mathrm{D}(P=.017$, after 50 minutes of clamping), group $\mathrm{D}>$ group $\mathrm{B}(P=.014$, after 45 minutes of clamping $)$, and group $\mathrm{B}>$ group $\mathrm{E}(P<.001$, after 40 minutes of clamping). Histopathologic findings did not necessarily correspond with hind-limb neurologic function.

Conclusions: Dextrorphan reduced the physical findings associated with ischemic spinal cord injury, and continuous intra-aortic infusion prolonged the safe clamping time significantly more than delivery by other routes.

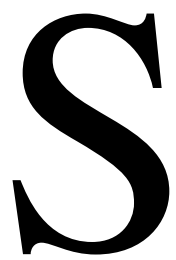

pinal cord ischemia is a severe complication of surgery on the descending aorta and thoracoabdominal aorta that has a major effect on the postoperative quality of life. ${ }^{1,2}$ Once the spinal cord becomes ischemic, the excitatory neurotransmitter glutamate is released from neuronal synapses and activates intracellular $\mathrm{Ca}^{2+}$ influx. This $\mathrm{Ca}^{2+}$ influx is thought to trigger a chain of reactions that lead to neuronal death. ${ }^{3}$ Protection of the central nervous system with noncompetitive antagonists of $N$ methyl-D-aspartate (NMDA), a glutamate receptor subtype, has been documented. ${ }^{4,5}$

Many drugs, including, dextromethorphan, ${ }^{6}$ dextrorphan, ${ }^{7-9}$ memantine, ${ }^{10}$ and riluzole, ${ }^{11}$ are known as noncompetitive NMDA receptor antagonists and have been shown to reduce paraplegia after spinal cord ischemia. Dextrorphan has been shown to protect the spinal cord against $15^{8}$ to $25^{9}$ minutes of ischemia. However, no study 

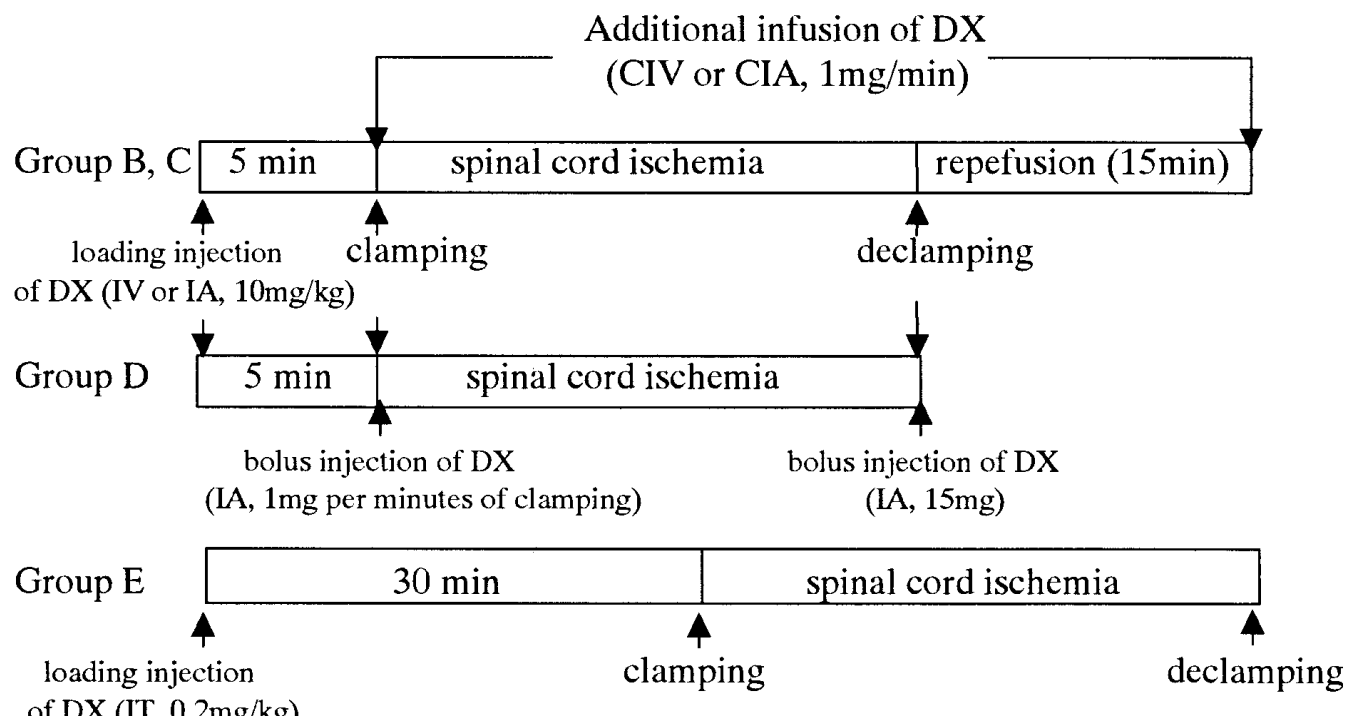

of DX (IT, $0.2 \mathrm{mg} / \mathrm{kg})$

Figure 1. Schematic representation of experimental protocol. Group A received simple infrarenal aortic clamping; group B, continuous intravenous infusion of dextrorphan; group C, continuous intra-aortic infusion of dextrorphan; group D, bolus intra-aortic injection of dextrorphan; and group $E$, bolus intrathecal injection of dextrorphan. $D X$, Dextrorphan; $I V$, intravenous infusion; $I A$, intra-aortic infusion; $C I V$, continuous intravenous infusion; CIA, continuous intra-aortic infusion; $I T$, intrathecal injection.

demonstrating the maximum length of the safe clamping time achievable by dextrorphan has been reported. We examined the effect of dextrorphan on spinal cord ischemic injury and sought to prolong the safe aortic clamping time by testing various drug delivery methods, such as intravenous infusion, intra-aortic injection, and intrathecal injection.

\section{Materials and Methods}

\section{Animal Care and Surgical Procedure}

We used male New Zealand White rabbits weighing 2.5 to $3.5 \mathrm{~kg}$. All animals received humane care in compliance with the "Guide for the Care and Use of Laboratory Animals" prepared by the Institute of Laboratory Animal Resources, National Research Council, and published by the National Academy Press, revised 1996, and the "Guidelines for Animal Experimentation" of Hamamatsu University School of Medicine.

Animals were randomly divided into 5 groups. Group A $(\mathrm{n}=$ 15) received simple infrarenal aortic clamping, group $B(n=20)$ received continuous intravenous infusion of dextrorphan, group $C$ $(\mathrm{n}=35)$ received continuous intra-aortic infusion of dextrorphan, group $\mathrm{D}(\mathrm{n}=25)$ received a bolus intra-aortic injection of dextrorphan, and group $E(n=15)$ received a bolus intrathecal injection of dextrorphan. Each dextrorphan-treated group had a small group of control animals $(\mathrm{n}=5$ each), which were given an equal amount of normothermic $\left(24^{\circ} \mathrm{C}\right)$ saline solution for 30 minutes after clamping. The rabbits were anesthetized with intravenous sodium pentobarbital $(25 \mathrm{mg} / \mathrm{kg})$. Intravenous vecuronium bromide $(0.1$ $\mathrm{mg} / \mathrm{kg}$ ) was also used in group $\mathrm{E}$ as a muscle relaxant to avoid movement during needle insertion into the spinal canal. The ani- mals were allowed to breathe spontaneously except in group E, in which tracheotomy and mechanical ventilation were necessitated by use of the muscle relaxant. All groups received $0.5 \%$ lidocaine hydrochloride at the site of skin incision as local anesthesia. After the incision had been made in the neck, an arterial line was secured in the left common carotid artery with a 24-gauge catheter for monitoring of the arterial pressure proximal to the site of clamping. A continuous intravenous infusion line was secured in the left external jugular vein with a 24-gauge catheter. A continuous intraaortic infusion line was established in the right femoral artery with a 20-gauge catheter, the tip of which extended to the infrarenal abdominal aorta. Rectal temperature was continuously monitored with a flexible probe (Terumo Medical Corporation, Tokyo, Japan). The animal was placed in the supine position and the infrarenal abdominal aorta was exposed through a transperitoneal approach. After intravenous or intra-aortic administration of heparin $(100 \mathrm{U} / \mathrm{kg})$, spinal cord ischemia was induced by crossclamping the abdominal aorta just distal to the origin of the renal arteries and just proximal to the aortic bifurcation. Each of the 5 groups was subdivided into a number of separate cohorts on the basis of the duration of clamping time. The subgroups, which consisted of 5 animals each, were constructed in the following way: group A, at 20, 25, and 30 minutes of clamping; group B, every 5 minutes from 30 to 45 minutes of clamping; group C, every 5 minutes from 30 to 60 minutes of clamping; group D, every 5 minutes from 30 to 50 minutes of clamping; and group E, at 25, 30, and 40 minutes of clamping.

\section{Drug Delivery}

Dextrorphan (Hoffman-La Roche Inc, Basel, Switzerland) was dissolved in saline solution at a concentration of $10 \mathrm{mg} / \mathrm{mL}$ for the treat- 


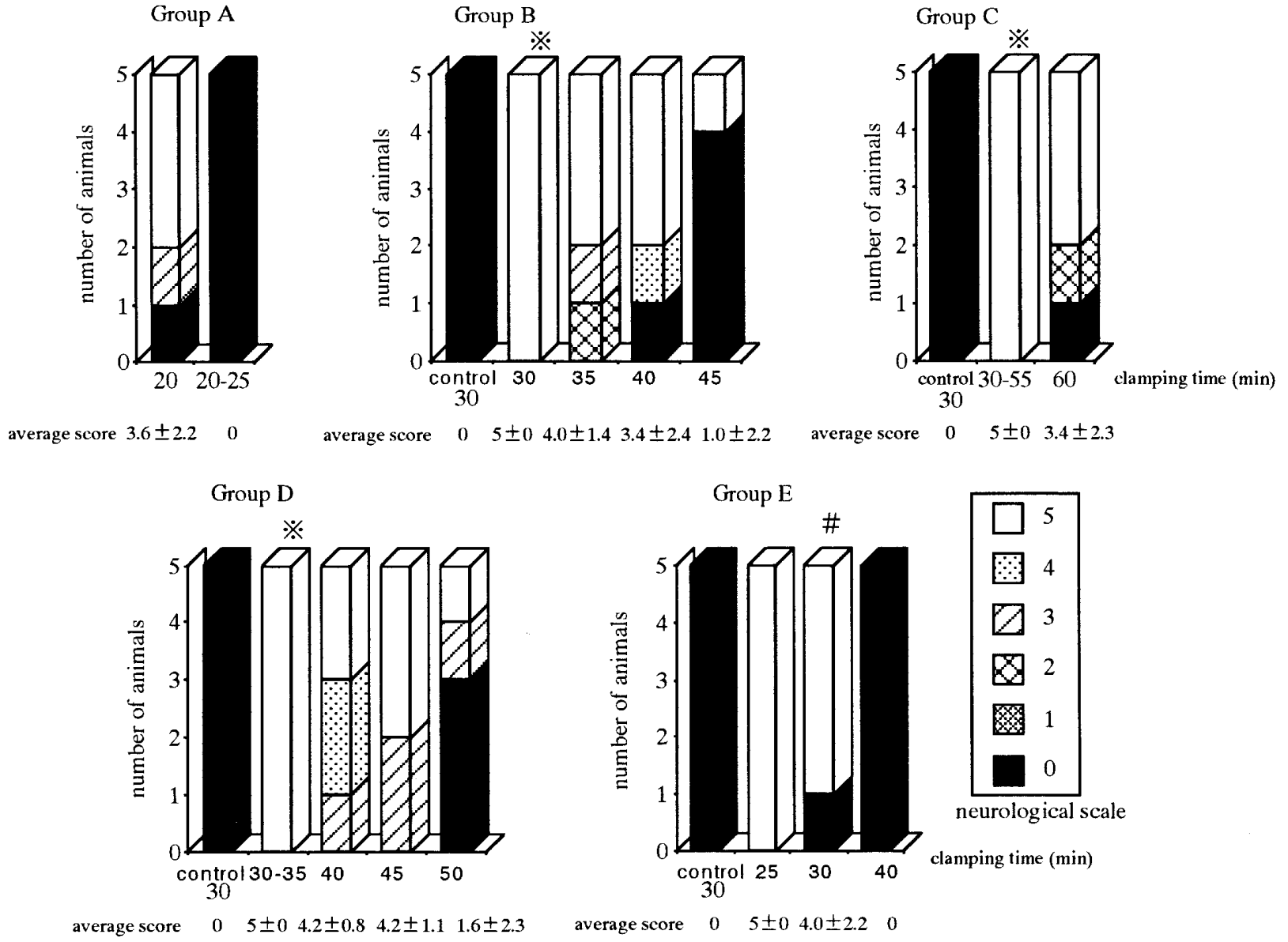

Figure 2. Neurologic status 48 hours after unclamping as evaluated by the Johnson neurologic recovery scale. Average scores represent mean \pm SD. The figures $20-25,30-55$, and 30-35 shown along the axis of the graph of groups A, C, and D indicate neurologic outcome every 5 minutes from 20 to 25 minutes, from 30 to 55 minutes, and from 30 to 35 minutes of clamping, respectively. ${ }^{*} P<.001$ versus control after 30 minutes of clamping. \#P=.014 versus control after 30 minutes of clamping.

ment of groups B, C, and D. For group E, a $1-\mathrm{mg} / \mathrm{mL}$ concentration of dextrorphan was prepared. In group $\mathrm{B}$, dextrorphan pretreatment $(10 \mathrm{mg} / \mathrm{kg})$ was done through the external jugular vein catheter $5 \mathrm{~min}-$ utes before aortic clamping. This was followed by continuous intravenous infusion of dextrorphan at a rate of $1 \mathrm{mg} / \mathrm{min}$ with an infusion pump (Terumo) until 15 minutes after unclamping. Group $C$ received continuous intra-aortic infusion of dextrorphan into the closed segment of the abdominal aorta after pretreatment $(10 \mathrm{mg} / \mathrm{kg})$ through the catheter in the right femoral artery 5 minutes before aortic clamping. Group D had similar dextrorphan pretreatment to group $\mathrm{C}$ and then received a bolus injection of dextrorphan $(1 \mathrm{mg}$ per minute of clamping time) into the closed segment of the aorta through the same route as group $\mathrm{C}$ immediately after clamping, as well as an additional $15 \mathrm{mg}$ of dextrorphan after unclamping. In group E, a tracheotomy was done to start mechanical ventilation. Then laminectomy was performed via a posterior approach in the left lateral position. The spinous processes of L5 were removed, and the surrounding structures were dissected until the epidural fat was exposed. A bolus dose of dextrorphan $(0.2 \mathrm{mg} / \mathrm{kg})$ was given intrathecally 30 minutes before spinal cord ischemia was induced (Figure 1).

\section{Neurologic Evaluation}

Neurologic status was scored by assessment of hind-limb motor function 48 hours after unclamping according to the Johnson neurologic recovery scale ${ }^{12}: 0=$ hind-limb paralysis; $1=$ severe paraparesis; 2 = functional movement, no hopping; 3 = ataxic hopping; $4=$ minimal ataxia; $5=$ normal.

\section{Histopathologic Examination}

After these assessments, animals were put to death with an overdose of intravenous sodium pentobarbital. The lumbar spinal cord (L4 to L6) was quickly removed and fixed overnight in $10 \%$ buffered formalin. Paraffin-embedded spinal cord sections were processed for hematoxylin and eosin staining, and histopathologic evaluation, including a count of the remaining neurons per section in the gray matter, was performed under a light microscope.

\section{Statistical Analysis}

The average neurologic recovery score was calculated and was expressed as mean \pm standard deviation (SD). Statistical analysis of the neurologic recovery scores was performed with the non- 


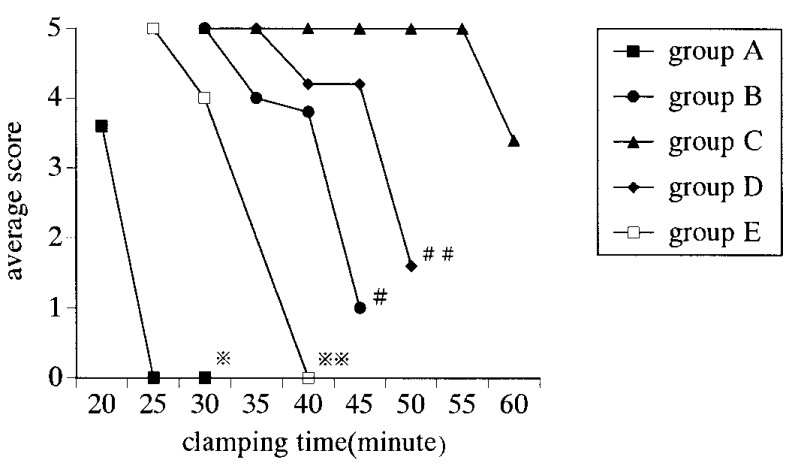

Figure 3. Average scores for the neurologic recovery scale. Standard deviations are eliminated. ${ }^{*} P<.001$ compared with groups $B, C$, and $D$ and $P=.014$ compared with group $E$ after 30 minutes of clamping. ${ }^{* *} P<.001$ compared with group $C, P=.005$ compared with group $D$, and $\boldsymbol{P}=.017$ compared with group $B$ after 40 minutes of clamping. $\# P=.014$ compared with group $C$ and $\boldsymbol{P}=.044$ compared with group $D$ after 45 minutes of clamping. \#\# $=.017$ compared with group $C$ after 50 minutes of clamping.

parametric Mann-Whitney $U$ test. Statistical analyses of the mean proximal arterial pressure, rectal temperature data, and the numbers of the remaining neurons were done with the unpaired $t$ test.

\section{Results}

Neurologic Evaluation

Figure 2 represents the neurologic outcome in all groups. The neurologic scores in group A were $3.6 \pm 2.2,0$, and 0 after 20, 25, and 30 minutes of clamping, respectively. All control animals in groups B, C, D, and E subjected to 30 minutes of clamping showed paraplegia (score 0 ). The neurologic scores for dextrorphan-treated animals in group B were 5.0 after 30 minutes of clamping, whereas they were 4.0 $\pm 1.4,3.4 \pm 2.4$, and $1.0 \pm 2.2$ after 35,40 , and 45 minutes of clamping, respectively. The neurologic score for dextrorphantreated animals in group $\mathrm{C}$ was 5.0 after 30, 35, 40, 45, 50, and 55 minutes of clamping, and the score was $3.3 \pm 2.5$ after 60 minutes of clamping. The neurologic score for dextrorphan-treated animals in group D was 5.0 after 30 and 35 minutes of clamping, and the scores were $4.2 \pm 0.8,4.2 \pm 1.1$, and $1.6 \pm 2.3$ after 40,45 , and 50 minutes of clamping, respectively. The neurologic scores for dextrorphan-treated animals in group D were 5.0 after 25 minutes of clamping and $4.0 \pm$ 2.2 and 0 after 30 and 40 minutes of clamping, respectively.

\section{Comparison of Average Scores}

Each dextrorphan-treated group showed better neurologic function than their respective control group of animals after 30 minutes of clamping $(P<.001$ vs groups $\mathrm{B}, \mathrm{C}$, and $\mathrm{D} ; P$ $=.014$ vs group E). Groups B, C, and D showed better neurologic function than group $\mathrm{E}(P=.017$ vs group $\mathrm{B} ; P<$ .001 vs group $\mathrm{C} ; P=.005$ vs group $\mathrm{D})$ after 40 minutes of clamping. In addition, groups $\mathrm{C}$ and $\mathrm{D}$ showed better neu- rologic function than group $\mathrm{B}(P=.014$ vs group $\mathrm{C} ; P=$ .044 vs group D) after 45 minutes of clamping. Furthermore, group $\mathrm{C}$ showed better neurologic function than group D after 50 minutes of clamping $(P=.017)$. Therefore, among the dextrorphan-treated animals, those from group $\mathrm{C}$ showed the best neurologic function, and continuous intraaortic infusion of dextrorphan prolonged the safe aortic clamping time to 55 minutes (Figure 3 ).

\section{Blood Pressure and Rectal Temperature}

The mean arterial pressure was not affected by dextrorphan or by the method of administration, except that group $\mathrm{E}$ showed hypotension at 30 minutes $(P<.05$ vs groups $\mathrm{A}$ and $\mathrm{D} ; P<.001$ vs group $\mathrm{C})$ and 40 minutes $(P<.01$ vs group C) after clamping (Table 1). Rectal temperature decreased gradually after induction of anesthesia and continued to fall during aortic clamping because we did not use heating pads. There were no statistically significant differences among the groups, except that group E showed a significant decrease of rectal temperature at the point of aortic clamping and also after 10 minutes $(P<.01$ vs groups $\mathrm{A}, \mathrm{C}$, and $\mathrm{D} ; P<.05$ vs group $\mathrm{B}), 20$ minutes $(P<.05$ vs groups $\mathrm{A}, \mathrm{B}$, and $\mathrm{C})$, and 30 minutes of clamping $(P<.05$ vs groups $\mathrm{A}$ and $\mathrm{B})$.

\section{Histopathologic Evaluation}

The spinal cords of animals that recovered fully (score 5) after dextrorphan treatment were histologically well preserved with normal-looking motor neurons, which had clear nucleoli and Nissl substance on hematoxylin and eosin staining of cross sections of the lumbar spinal cord. On the other hand, some spinal cords from the animals with hindlimb paralysis (score 0) demonstrated necrotic changes, including destruction and vacuolization of the gray matter, pyknosis of neurons, and eosinophilic changes of the cytoplasm, but others appeared almost normal. However, it was difficult to detect any obvious differences in the histologic features of spinal cords with scores between 2 and 4 (Figure 4 ). The counts of remaining neurons apparently corresponded to the average neurologic scores (Figure 5).

\section{Discussion}

Ischemic neuronal death in the spinal cord and in the brain occurs because excessive glutamate release activates the glutamate NMDA receptor and triggers intracellular $\mathrm{Ca}^{2+}$ influx. ${ }^{13}$ The efficacy of noncompetitive NMDA receptor antagonists for prevention of neuronal ischemia has been documented, especially in the brain.,5 Similar studies on the spinal cord have also been conducted recently through intravenous, ${ }^{6,7,9-11}$ intra-aortic,,${ }^{9,10}$ and intraperitoneal routes. ${ }^{8}$

Dextrorphan, the $O$-demethylated metabolite of the common antitussive dextromethorphan, is an NMDA antagonist that attenuates hypoxic neuronal injury in cultured cells and significantly reduces ischemic neuronal injury in animal 

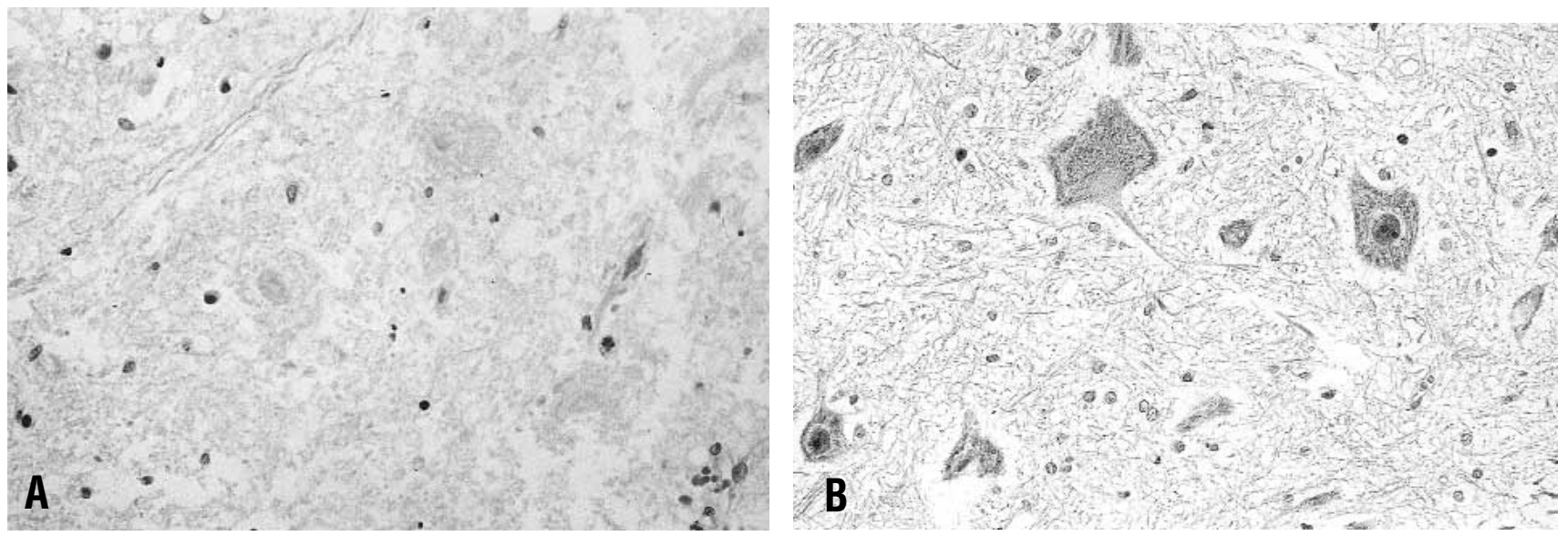

Figure 4. Representative photomicrographs of lumbar spinal cord sections from animals that received bolus intraaortic injection of dextrorphan and 50 minutes of clamping. Sections were stained with hematoxylin and eosin 48 hours after unclamping (original magnification 100x). The animals had hind-limb paralysis. Some spinal cords show necrotic changes, including destruction and vacuolization of the gray matter, pyknosis of neurons, and eosinophilic changes of the cytoplasm (A), and others show normal-looking motor neurons (B).

TABLE 1. Mean proximal arterial pressure (mm $\mathrm{Hg}$ ) during aortic clamping

\begin{tabular}{|c|c|c|c|c|c|c|c|}
\hline & \multicolumn{7}{|c|}{ Clamping time (min) } \\
\hline & Before & 10 & 20 & 30 & 40 & 50 & 60 \\
\hline Group A & $91 \pm 6$ & $90 \pm 8$ & $88 \pm 8$ & $87 \pm 9$ & & & \\
\hline Group B & $94 \pm 11$ & $94 \pm 9$ & $85 \pm 9$ & $85 \pm 8$ & $89 \pm 9$ & & \\
\hline Group C & $92 \pm 8$ & $89 \pm 10$ & $89 \pm 10$ & $90 \pm 10$ & $90 \pm 10$ & $90 \pm 10$ & $90 \pm 7$ \\
\hline Group D & $95 \pm 11$ & $89 \pm 11$ & $89 \pm 11$ & $85 \pm 11$ & $82 \pm 12$ & $89 \pm 14$ & \\
\hline Group E & $89 \pm 12$ & $85 \pm 13$ & $85 \pm 11$ & $75 \pm 9^{*}$ & $77 \pm 13 \dagger$ & & \\
\hline
\end{tabular}

Values represent mean \pm standard deviation. Group $A(n=15)$ received simple infrarenal aortic clamping; group $B(n=20)$, continuous intravenous infusion of dextrorphan; group $C(n=35)$, continuous intra-aortic infusion of dextrorphan; group $D(n=25)$, bolus intra-aortic injection of dextrorphan; and group $E(n=15)$, bolus intrathecal injection of dextrorphan.

${ }^{*} P<.05$ versus groups $A$ and $D ; P<.001$ versus group $C$.

$\dagger P<.01$ versus group $\mathrm{C}$.

stroke models. ${ }^{14}$ Pharmacokinetic studies have indicated that intravenous dextrorphan has a mean half-life of 1.7 to 5.4 hours. ${ }^{15}$ Dextromethorphan is rapidly converted to dextrorphan, which is 5 times more effective against hypoxic neuronal injury. ${ }^{16}$ In this study, dextrorphan was administered by means of 4 different methods: continuous intravenous infusion, continuous intra-aortic infusion, bolus intra-aortic injection, and bolus intrathecal injection. Each of the 5 groups was divided into separate cohorts according to their duration of clamping. At first, neurologic outcome at 30 minutes of clamping was evaluated and then clamping time was gradually lengthened at an increment of 5 minutes (group B, C, and D) or 10 minutes at a time (group E) until the neurologic status of the animals clearly deteriorated. Control animals in all groups except group A became paraplegic at 30 minutes of clamping. It was observed that the dextrorphan-treated animals of groups $\mathrm{B}, \mathrm{C}$, and D could safely withstand crossclamping for up to 30 minutes, whereas this duration for group $\mathrm{E}$ was 25 minutes. In our study, dextrorphan was effective irrespective of the method of administration when compared with control animals. Continuous intra-aortic infusion was found to be the most effective method, and it prolonged the safe clamping time up to 55 minutes. Bolus intra-aortic injection was found to be a little more effective than continuous intravenous infusion. The reason for this efficacy probably is that intraaortic dextrorphan is delivered more effectively to the ischemic spinal cord. However, the extent of neurologic recovery showed some variation in both groups. Continuous intra-aortic infusion led to a better outcome than bolus intraaortic injection, possibly because most of the dextrorphan injected in one shot was washed away from spinal cord. In 


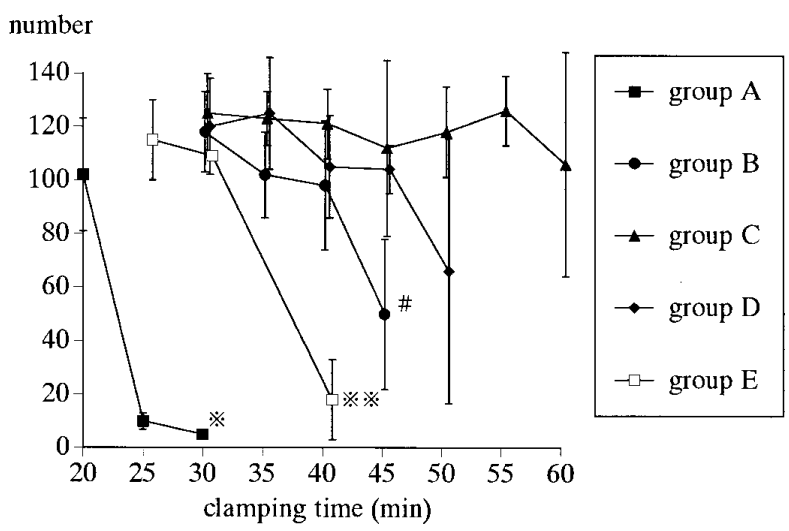

Figure 5. Neurons stained with hematoxylin and eosin in the gray matter of lumbar spinal cord. ${ }^{*} \boldsymbol{P}<.001$ versus other groups. ${ }^{* *} P<$ .001 versus other groups except group $A$. $\# P=0.013$ versus group $C$, and $P=.009$ versus group $D$.

this sense, bolus intra-aortic injection is similar to continuous intravenous infusion. It has also been demonstrated that dextrorphan must be continuously infused for adequate neuroprotection, because the drug failed to protect against ischemic edema or neuronal damage when given as a loading dose only, whereas a loading dose plus maintenance infusion showed significant protection. ${ }^{17}$ In our study, bolus intrathecal injection of dextrorphan was also effective against spinal cord ischemia, but the safe clamping time was not as long as we expected. Dextrorphan easily passes through the blood-brain barrier, and the blood level correlates well with the free brain level and with the extent of protection against ischemic neuronal damage. ${ }^{18}$ Thus, dextrorphan may infiltrate the entire spinal cord more effectively through the blood vessels than through the spinal fluid. Continuous intrathecal infusion of dextrorphan may prolong the safe clamping time, but it is difficult to insert an intrathecal catheter. Several studies have been performed with regard to percutaneous intrathecal injection in rats ${ }^{19}$ and rabbits. ${ }^{20}$ However, we found that percutaneous intrathecal injection in rabbits was difficult because the intrathecal space is very narrow. Blood pressure was stable in all groups while the aorta was crossclamped.

Hypothermia can inhibit glutamate release ${ }^{21}$ and prolong tolerance of ischemia. ${ }^{22}$ In this study, ischemia was induced with normothermia. The temperature of all groups except for the intrathecal group decreased spontaneously but was not significantly lower than that of group A. Therefore, hypothermia, which may have influenced the duration of the safe clamping time, did not play a major role in protecting the spinal cord from ischemia. Although the body temperature of the intrathecal group was significantly lower than in other groups, this may be attributable to the preischemic fall of temperature in this group. The reason for the preischemic hypothermia was that the preischemic operating time was longer in the intrathecal group because of additional invasive procedures. However, the rate of decrease in body temperature in this group was similar to that in the other groups.

Histopathologic findings did not necessarily correspond well with hind-limb neurologic function. Even the spinal cords of paraplegic animals were sometimes histologically normal. These results suggest that the mechanism of motor neuron deficits is complex and that the spinal cords of paraplegic animals do not always show obvious necrosis. We counted only the remaining neurons because necrotic neurons, which lost nuclear hematoxylin, were difficult to distinguish from surrounding tissue. Ortiz and associates ${ }^{23}$ described cytopathologic changes in the cingulate cortex of rats treated with a $30-\mathrm{mg} / \mathrm{kg}$ dose of dextrorphan, and these animals did not exhibit overt changes such as ataxia. The present study, however, revealed no obvious histopathologic changes in the animals that did not show any neurologic deficits.

It has also been reported that transient and reversible adverse effects, including nystagmus, nausea, vomiting, somnolence, hallucinations, agitation, and hypotension at high doses, can occur in dextrorphan-treated patients with acute stroke. ${ }^{15}$ However, such adverse effects resolve rapidly after discontinuation of infusion because of the short half-life of the drug, and dextrorphan does not exhibit any cardiac or other systemic toxicities. ${ }^{15}$ No findings obviously attributable to drug toxicity were noted in this study. However, in the clinical situation attention should be paid to such a possibility. It was demonstrated that preoperative ${ }^{8,9}$ and postoperative treatment ${ }^{17,18}$ with dextrorphan improved the tolerance of the spinal cord to ischemia. Improvement in neurologic outcome might also be expected in the clinical situation because pretreatment of patients undergoing thoracic and thoracoabdominal aortic operations is possible.

Segmental sequential repair and distal aortic perfusion with the aid of partial cardiopulmonary bypass is performed in many hospitals including ours as a standard surgical treatment for thoracoabdominal aortic aneurysms to shorten the spinal ischemic time as much as possible. ${ }^{24-26}$ Hypothermic circulatory arrest has been used when the location, extent, or severity of aortic disease precludes placement of clamps on the aorta. ${ }^{27}$ Nevertheless, postoperative neurologic deficits are difficult to prevent completely, and supplementary procedures might be required. A multimodality protocol described by Hollier and associates, ${ }^{28}$ which included cerebrospinal fluid drainage, maintenance of proximal hypertension during crossclamping, and the use of mannitol, steroids, and calcium channel blockers, appeared to be effective in reducing the risk of spinal cord injury during thoracoabdominal aortic replacement. We think the use of neuroprotective drugs might add to the options in this regard. We assume that continuous intravenous infusion of dextrorphan might be comparable with continuous distal aortic perfusion (which is used for cardiopulmonary bypass) because dextrorphan is 
distributed systemically except in the crossclamped aortic segment. Therefore, intravenous dextrorphan infusion is also expected to prolong the safe clamping time. A thorough application of our experimental procedure of continuous intra-aortic infusion to the thoracoabdominal operation in human beings is impossible. However, for the clinical use of this continuous intra-aortic infusion method, selective intercostal arterial perfusion, either through a small Dacron prosthesis ${ }^{29}$ or through catheters of $1-\mathrm{mm}$ or $1.5-\mathrm{mm}$ diameter, ${ }^{30}$ might be helpful in achieving continuous infusion directly into the intercostal arteries of the crossclamped aortic segment. However, insertion of such narrow catheters is often time consuming, technically difficult, and associated with risk of injury to the intercostal arteries. Continuous intrathecal infusion via a catheter was impossible in rabbits, but such intrathecal catheterization can be done percutaneously in human beings. The procedure is likely to be more effective if two catheters are used, one for infusion and the other for drainage.

The safe clamping time was prolonged in all of the dextrorphan-treated animals when compared with the control animals. Continuous intra-aortic infusion of dextrorphan, in particular, was able to prolong the safe aortic clamping time, which was significantly longer than that provided by any other route. The present experiment was done in an infrarenal aortic clamping model rather than a thoracoabdominal aortic clamping model. However, the results of this study suggest that dextrorphan has the potential to reduce spinal cord ischemia in patients undergoing thoracoabdominal aortic operations. Further clinical investigations are required to determine the beneficial effects of dextrorphan.

\section{References}

1. Svensson LG, Crawford ES, Hess KR, Coselli JS, Safi HJ. Experience with 1509 patients undergoing thoracoabdominal aortic operation. $J$ Vasc Surg. 1993; 17:357-70.

2. Coselli JS. Thoracoabdominal aortic aneurysm: experience with 372 patients. J Card Surg. 1994;9:638-47.

3. Choi DW, Maulucci-Gedde M, Kriegstein AR. Glutamate neurotoxicity in cortical cell culture. J Neurosci. 1987;7:357-68.

4. Simon RP, Swan JH, Griffiths T, Meldrum BS. Blockade of $N$-methyl$\mathrm{D}$-aspartate receptors may protect against ischemic damage in the brain. Science. 1984;226:850-2.

5. Kochhar A, Zivin JA, Lyden PD, Mazzarella V. Glutamate antagonists therapy reduces neurologic deficits produced by focal central nervous system ischemia. Arch Neurol. 1988;45:148-53.

6. George CP, Goldberg MP, Choi DW, Steinberg GK. Dextromethorphan reduces neocortical ischemic neuronal damage in vivo. Brain Res. 1988;440:375-9.

7. Rokkas CK, Helfrich LR, Lobner DC, Choi DW, Kouchoukos NT. Dextrorphan inhibits the release of excitatory amino acids during spinal cord ischemia. Ann Thorac Surg. 1994;58:312-20.

8. Kato H, Kanellopoulos GK, Matsuo S, Wu YJ, Jacquin MF, Hsu CY, et al. Protection of rat spinal cord from ischemia with dextrorphan and cycloheximide: effects on necrosis and apoptosis. $J$ Thorac Cardiovasc Surg. 1997;114:609-18.

9. Nakamura M. Protection from spinal cord ischemia during aortic repair using a noncompetitive $N$-methyl-D-aspartate receptor antagonist. Sapporo Med J. 1996;65:179-88.

10. Ehrlich M, Knolle E, Ciovica R, Bock P, Turkof E, Grabenwöger M, et al. Memantine for prevention of spinal cord injury in a rabbit model. J Thorac Cardiovasc Surg. 1999;117:285-91.

11. Lang-Lazdunski L, Heurteaux C, Vaillant N, Widmann C, Lazdunski M. Rilusole prevents ischemic spinal cord injury caused by aortic crossclamping. J Thorac Cardiovasc Surg. 1999;117:881-9.

12. Johnson SH, Kraimer JM, Graeber GM. Effects of flunarizine on neurological recovery and spinal cord blood flow in experimental spinal cord ischemia in rabbits. Stroke. 1993;24:1547-53.

13. Simpson RK, Robertson CS, Goodman JC. Spinal cord ischemiainduced elevation of amino acids: extracellular measurement with microdialysis. Neurochem Res. 1990;15:635-9.

14. Choi DW. Dextrorphan and dextromethorphan attenuate glutamate neurotoxicity. Brain Res. 1987;403:333-6.

15. Albers GW, Atkinson RP, Kelley RE, Rosenbaum DM. Safety, tolerability, and pharmacokinetics of the $N$-methyl-D-aspartate antagonist dextrorphan in patients with acute stroke. Stroke. 1995;26:254-8.

16. Church J, Lodge D, Berry SC. Differential effects of dextrorphan and levorphanol on the excitation of rat spinal neurons by amino acids. Eur J Pharmacol. 1985;111:185-90.

17. Faden AI, Ellison JA, Noble LJ. Effects of competitive NMDA receptor antagonists in spinal cord injury. Eur J Pharmacol. 1990;175:165-74.

18. Breckwoldt WL, Genco CM, Connolly RJ, Cleveland RJ, Diehl JT. Spinal cord protection during aortic occlusion: efficacy of intrathecal tetracaine. Ann Thorac Surg. 1991;51:959-63.

19. Steinberg GK, Yoon EJ, Kunis DM, Sun GH, Maier CM, Grant GA. Neuroprotection by $N$-methyl-D-aspartate antagonists in focal cerebral ischemia is dependent on continued maintenance dosing. Neuroscience. 1995;64:99-107.

20. Steinberg GK, Kunis D, Saleh J, De La Paz R. Protection after transient focal cerebral ischemia by the $N$-methyl-D-asparatate antagonist dextrorphan is dependent upon plasma and brain levels. J Cereb Blood Flow Metab. 1991;11:1015-24.

21. Busto R, Globus MY, Dietrich WD, Martine E, Valdes I, Ginsberg MD. Effect of mild hypothermia on ischemia-induced release of neurotransmitters and free fatty acids in rat brain. Stroke. 1989;20:904-10.

22. Mitani A, Kataoka K. Critical levels of extracellular glutaminate mediating gerbil hippocampal delayed neuronal death during hypothermia: brain microdialysis study. Neuroscience. 1991;42:661-70.

23. Ortiz GG, Guerrero JM, Reiter RJ, Poeggeler BH, Bitzer-Quintero OK, Feria-Velasco A. Neurotoxicity of dextrorphan. Arch Med Res. 1999;30:125-7.

24. Kazui T, Komatsu S, Yokoyama H. Surgical treatment of aneurysms of the thoracic aorta with the aid of partial cardiopulmonary bypass: an analysis of 95 patients. Ann Thorac Surg. 1987;43:622-7.

25. Kazui T, Komatsu S, Sasaki T, Yamada O. Graft inclusion technique for thoracoabdominal aortic aneurysms involving visceral branches with the aid of a femoro-femoral bypass. J Cardiovasc Surg. 1987; 28:663-70.

26. Safi HJ, Hess KR, Randel M, Iliopoulos DC, Baldwin JC, Mootha R, et al. Cerebrospinal fluid drainage and distal aortic perfusion: reducing neurologic complications in repair of thoracoabdominal aortic aneurysm type I and II. J Vasc Surg. 1996;23:223-9.

27. Kouchoukos NT, Daily BB, Rokkas CK, Murphy SF, Bauer Sabboud $\mathrm{N}$. Hyperthermic bypass and circulatory arrest for operations on the descending thoracic and thoracoabdominal aorta. Ann Thorac Surg. 1995;60:67-77.

28. Hollier LH, Money SR, Naslund TC, Proctor CD, Buhrman WC, Marino RJ. Risk of spinal cord dysfunction in patients undergoing thoracoabdominal aortic replacement. Am J Surg. 1992;164:210-3.

29. Sueda T, Morita S, Okada K, Orihashi K, Shikata H, Matsuura Y. Selective intercostal arterial perfusion during thoracoabdominal aortic aneurysm surgery. Ann Thorac Surg. 2000;70:44-7.

30. Ueda T, Shimizu H, Mori A, Kashima I, Moro K, Kawada S. Selective perfusion of segmental arteries in patients undergoing thoracoabdominal aortic surgery. Ann Thorac Surg. 2000;70:38-43. 